\title{
Insights from a failure of selective adaptation: Syllable-initial and syllable-final consonants are different
}

\author{
ARTHUR G. SAMUEL \\ Yale University, New Haven, Connecticut
}

\begin{abstract}
Selective adaptation with a syllable-initial consonant fails to affect perception of the same consonant in syllable-final position, and vice versa. One account of this well-replicated result invokes a cancellation explanation: with the place-of-articulation stimuli used, the pattern of formant transitions switches according to syllabic position, allowing putative phonetic-level effects to be opposed by putative acoustic-level effects. Three experiments tested the cancellation hypothesis by preempting the possibility of acoustic countereffects. In Experiment 1, the test syllables and adaptors were $/ \mathrm{r} /-\Lambda / \mathrm{CVs}$ and $\mathrm{VCs}$, which do not produce cancelling formant patterns across syllabic position. In Experiment 2, /b/-/d/ continua were used in a paired-contrast procedure, believed to be sensitive to phonetic, but not acoustic, identity. In Experiment 3, cross-ear adaptation, also believed to tap phonetic rather than acoustic processes, was used. All three experiments refuted the cancellation hypothesis. Instead, it appears that the perceptual process treats syllableinitial consonants and syllable-final ones as inherently different. These results provide support for the use of demisyllabic representations in speech perception.
\end{abstract}

A basic premise of an information-processing approach to speech perception is that the speech signal undergoes a series of transformations. Each successive transformation encodes the information in a form that is further removed from its initial transduction of the acoustic pattern. In this framework, a fundamental issue is the form of representation that results from each transformation.

Various different kinds of representations have been suggested by speech researchers. In the early processing of speech, many investigators have suggested that two qualitatively different levels of analysis are necessary to account for a variety of experimental results. Most of these researchers distinguish between an initial auditory representation and a phonetic one (e.g., Fujisaki \& Kawashima, 1969; Pisoni, 1973; Sawusch, 1977a). Some investigators prefer to distinguish between relatively simple, untransformed auditory representations and more complex, abstract ones (Kat \& Samuel, 1984; Samuel, 1986; Samuel \& Newport, 1979); one variation accommodates two levels of auditory abstraction, and a phonetic one (Sawusch, 1986). Despite these differences in details of the models, there is actually surprisingly good agreement that two levels of analysis are needed (see, e.g., Eimas \& Miller, 1978; Jamieson \& Cheesman, 1986; Samuel, 1986; Sawusch, 1986; Simon \& StuddertKennedy, 1978).

Many of the studies that have led to this conclusion have employed the selective adaptation paradigm, first used in

Address correspondence to Arthur G. Samuel, Department of Psychology, Yale University, P.O. Box 11 A Yale Station, New Haven, CT 06520-7447. speech research by Eimas and Corbit (1973). The technique, borrowed from visual psychophysics (e.g., Blakemore \& Sutton, 1969), involves the repetitive presentation of a stimulus in order to induce changes in the perception of related stimuli. For example, Eimas and Corbit synthesized a continuum of syllables in which one endpoint sounded like /ba/ and the other sounded like /pa/; stimuli near the middle of the continuum were somewhat ambiguous. After listening to repeated presentation of $/ \mathrm{ba} /$, listeners identified fewer syllables as $/ \mathrm{ba} /$ than before the adaptation. Similarly, the repeated presentation of /pa/ reduced /pa/ reports. Perhaps more interesting was the effect of related adaptors: /da/ (which is voiced, like $/ \mathrm{ba} /$ ) reduced $/ \mathrm{ba} /$ report, and $/ \mathrm{ta} /$ (unvoiced, like $/ \mathrm{pa} /$ ) reduced $/ \mathrm{pa} /$ report.

These findings illustrate the utility of the adaptation paradigm for mapping the perceptual system's similarity space. Two stimuli that are treated as similar by the perceptual system may be expected to yield cross-adaptation (e.g., /da/ and $/ \mathrm{ta} /$ adpators on a $/ \mathrm{ba} /-/ \mathrm{pa} /$ test series); a lack of such adaptation may be taken as evidence for the perceptual dissimilarity of two stimuli.

Within this framework, a failure of adaptation, first reported by Ades (1974), is of particular interest. Ades found that /bae/ and /dae/ were effective adaptors on a /bae/-/dae/ test series, but that they were ineffective on an /aeb/-/aed/ continuum. Similarly, /aeb/ and /aed/ shifted labeling of /aeb/-/aed/, but not of /bae/-/dae/. The position-specificity of adaptation effects is robust, having been replicated by Miller and Eimas (1976), Pisoni and Tash (1975), Samuel, Kat, and Tartter (1984), Sawusch (1977b), and Wolfe (1978). By the logic outlined above, these results suggest that syllable-initial consonants 
are treated as dissimilar to syllable-final ones, even when they share phonemic identity.

There is an alternative interpretation of the null adaptation effect, suggested by Pisoni and Tash (1975), which can best be understood by reference to Figure 1 . This figure presents schematic spectrograms of $/ \mathrm{ab} /, \mathrm{/ba} /, / \mathrm{ad} /$, and $/ \mathrm{da} /$. The critical point to note is that the second and third formant transitions (which carry the critical information for perceiving place of articulation) are rising in $/ \mathrm{ba} /$ and $/ \mathrm{ad} /$ and falling in $/ \mathrm{da} /$ and $/ \mathrm{ab} /$. Pisoni and Tash suggested that the null cross-series effects might actually reflect the operation of two opposing effects: the usual phonetic (or second-level) adaptation effect of, for exam$\mathrm{ple}, / \mathrm{ba} / \mathrm{on} / \mathrm{ab} /-/ \mathrm{ad} /$ would be counteracted by an opposite acoustic (or first-level) effect (since the transitions of $/ \mathrm{ba} / \mathrm{match} / \mathrm{ad} /$, rather than $/ \mathrm{ab} /$ ).

The research to be reported here includes three experiments intended to distinguish between this cancellation hypothesis and the hypothesis that syllable-initial and syllable-final consonants are fundamentally dissimilar from the perceptual system's perspective. Experiment 1 contrasts these views by looking for cross-series effects using CV and VC stimuli whose transition structure is such that cancellation is not possible. Experiment 2 compares adaptation and paired-contrast effects on the cross-

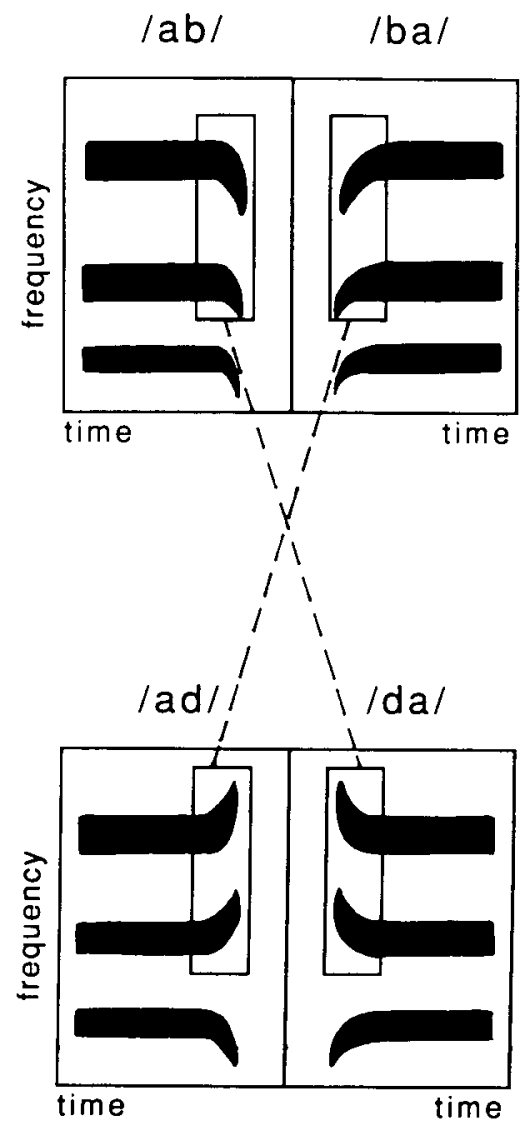

Figure 1. Schematic spectrograms of $/ \mathrm{ba} /, / \mathrm{da} / \mathrm{/ab} /$, and $/ \mathrm{ad} /$, showing matched parts of the second and third formant transitions. series case; the latter paradigm has been found to tap only second-level (phonetic) processing (see Samuel, 1986) and should therefore not be subject to cancellation. Finally, Experiment 3 compares ipsilateral (monaural) adaptation effects to contralateral (monaural) ones. Contralateral presentation of the adaptor and test items has been argued to tap central (second-level) processing, again without the possibility of low-level acoustic cancellation (see Samuel, 1986; Sawusch, 1977b). If these experiments find crossseries effects, we may infer that cancellation by lower level adaptation was masking these effects in the Ades (1974) study. If instead cross-series effects remain small or nonexistent, then we may conclude that there are separate representations of syllable-initial and syllablefinal consonants.

\section{EXPERIMENT 1}

As noted above, the basic noneffect of Ades (1974) has been well replicated. Surprisingly, all of the replications have used place-of-articulation stimuli for which the transition-reversal possibility diagrammed in Figure 1 is possible: Miller and Eimas (1976), Pisoni and Tash (1975), Samuel, Kat, and Tartter (1984), and Sawusch (1977b) used $/ \mathrm{b} /-/ \mathrm{d} /$ continua, and Wolfe (1978) used $/ \mathrm{d} /-/ \mathrm{g} /$ (where the third formant reverses according to syllabic position). Experiment 1 involved two continua that do not have this property: $/ \mathrm{ri} /-/ \mathrm{li} /$ and $/ \mathrm{ir} /-/ \mathrm{il} /$. As Figure 2 illustrates, the critical acoustic cue for the $/ \mathrm{r} /-/ \mathrm{l} /$ distinction is the slope of the third formant: / $r /$ has a steep transition, whereas /1/ has a shallow one. Note that although the direction of this transition changes with syllabic position (rising initially, falling finally), the relationship of transitions to phonemes does not change; the transitions for a syllable-initial /1/ do not in any way resemble those of a syllable-final $/ r /$. As such, no lowlevel cancellation of higher level adaptation effects should occur.

The $/ r /-/ 1 /$ stimuli were selected for two reasons beyond satisfying the "noncancellation" structure criterion. First, they are "encoded" stimuli, much like the stop consonants used in previous research. To find cross-series effects with, for example, an $/ \mathbf{s} /-/ \mathbf{s} /$ series might not speak to the cancellation issue, since such effects could be attributed to low-level invariant cues. Second, the $/ r /-/ 1 /$ stimuli used here are adapted from stimuli that have been shown to produce clean, categorical identification functions in both CV and VC form (McGovern \& Strange, 1977).

\section{Method}

Stimuli. The stimuli for Experiment 1 were two eight-step continua generated with the cascade branch of the Klatt (1980) software synthesizer. All syllables were $290 \mathrm{msec}$ long, including a 140 -msec consonantal portion and a 150 -msec vowel segment. The steady-state vowel formants were at $286 \mathrm{~Hz}$ (F1), $2234 \mathrm{~Hz}$ (F2), and $2862 \mathrm{~Hz}$ (F3). The fundamental frequency for the first $185 \mathrm{msec}$ of each syllable was $100 \mathrm{~Hz}$; it fell linearly to $85 \mathrm{~Hz}$ at syllable 


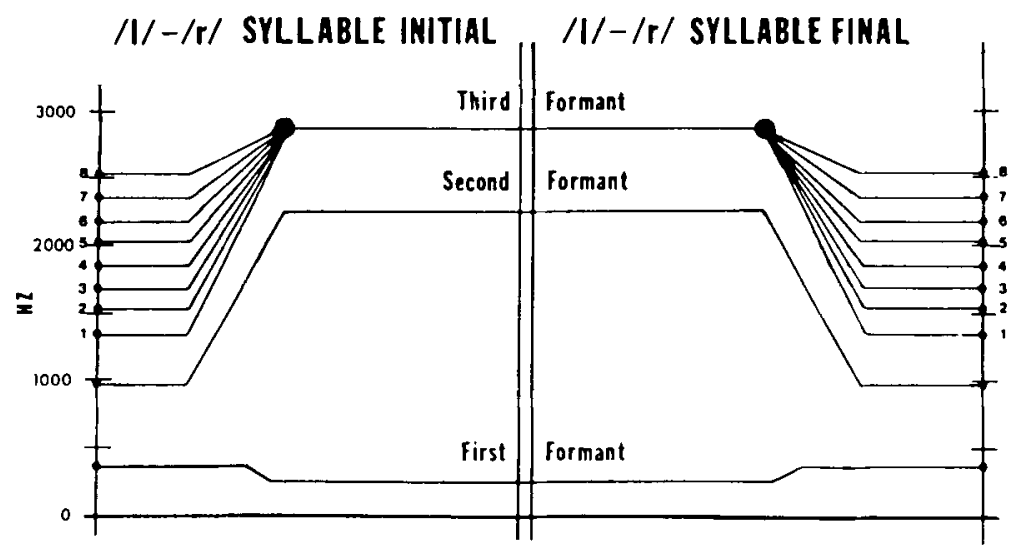

Figure 2. Schematic spectrogram of the /ri/-lli/ and /ir/-/il/ stimuli used in Experiment 1. (Based on Figure 1 in "The perception of $/ r /$ and $/ 1 /$ in syllable-initial and syllablefinal position," by K. McGovern \& W. Strange, Perception \& Pychophysics, 21, 162 170. Copyright 1977 by Psychonomic Society, Inc. Used by permission.)

offset. The amplitude of voicing was ramped from 45 to $55 \mathrm{~dB}$ over the initial $140 \mathrm{msec}$, and it was returned to $45 \mathrm{~dB}$ over the last $45 \mathrm{msec}$.

Each CV began with a 70 -msec steady-state portion, followed by 70 msec of transitions to the vowel; each VC had a corresponding mirror-image offset. The first-formant steady state, at $361 \mathrm{~Hz}$, lasted $115 \mathrm{msec}$; the F1 transition was $35 \mathrm{msec}$. The 70-msec F2 steady state was at $996 \mathrm{~Hz}$. The steady-state frequency of the third formant was varied in $170-\mathrm{Hz}$ steps, from $1340 \mathrm{~Hz}(/ \mathrm{r} /)$ to $2530 \mathrm{~Hz}$ (//). A 70-msec transition from each steady state reached the vowel's F3 of $2862 \mathrm{~Hz}$. Note that all CV F3 transitions rose, and all VC F3 transitions fell. This structure cannot produce the cancellation found with $/ b /-/ d /$ stimuli.

Apparatus and Procedure. The subjects participated in four 1-h sessions held on consecutive days. Each session included two tests: a baseline identification test and an adaptation test. The baseline ID consisted of 20 randomizations of the 16 stimuli (8 CV and 8 VC). The first 2 passes were practice and were not scored. The subjects were instructed to identify the consonant in each syllable as " $L$ " or " $R$," using labeled response keys. Accuracy was stressed primarily, speed secondarily. The adaptation test included 18 passes through the 16 stimuli. Identification trials in blocks of 8 stimuli were interspersed with 30 -sec adaptation periods (45 iterations). An initial 60 -sec adaptation period (90 iterations) preceded the rest of the adaptation test. During adaptation periods, the subjects were instructed to simply listen to the repeating syllable. During identification blocks they were told to respond as in the identification test.

The two CV endpoints and two VC endpoints were used as the adaptors. The order of adaptation conditions was counterbalanced, using a Latin square design.

Adaptors and test items were stored on disk files on a PDP-11/73 computer. For presentation to the subjects, the stimuli were output through a D/A converter (12 bits), low-pass filtered (4.8 kHz), amplified, and presented binaurally over high-quality headphones. The timing of presentation and response was controlled by the PDP-11/73.

Subjects. Twelve subjects participated in Experiment 1. All were native English speakers with no known hearing problems. One subject, who failed to label the stimuli consistently, was replaced by another subject from the same population. The subjects were tested in groups of 3 in a sound-attenuating chamber. They were paid for their participation.

\section{Results and Discussion}

For each subject, the average percentage of $/ r /$ responses was computed. For analysis purposes, the average labeling of the middle four stimuli of each continuum was used as the summary statistic; adaptation shifts were indexed by subtracting the baseline value of this statistic from its value after adaptation.

The data are summarized in Figures 3 and 4 . Figure 3 shows the identification of $/ \mathrm{ri} /-/ \mathrm{li} /$ stimuli, and Figure 4 depicts the labeling of the /ir/-/il/ syllables. Consider first the within-position effects of syllable-initial consonants, shown in the top two panels of Figure 3. Both /ri/ and /li/ produced robust adaptation shifts: / $\mathrm{r} /$ reduced $/ \mathrm{r} /$

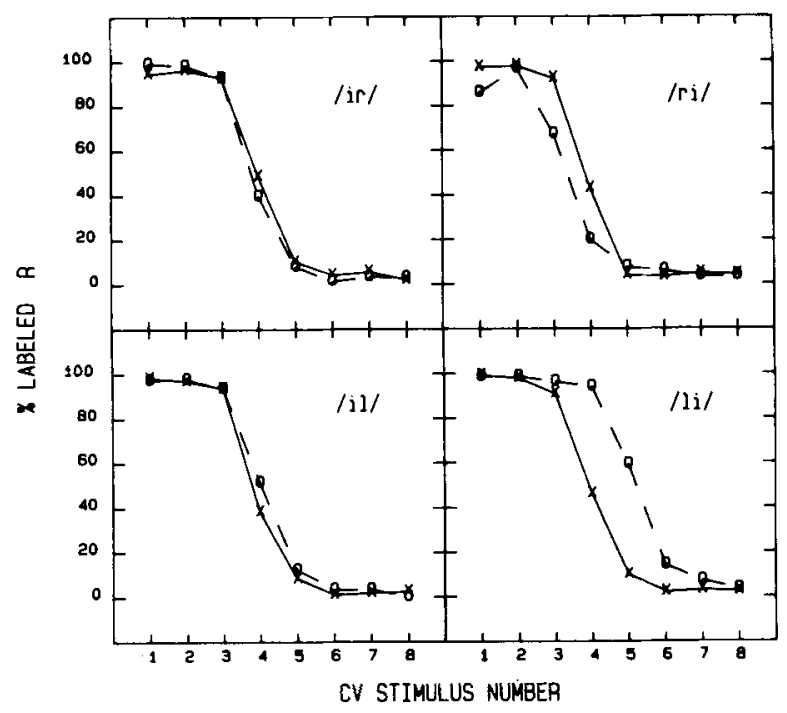

Figure 3. Identification functions for the /ri/-/li/ stimuli, before and after adaptation. Baseline labeling is shown with solid lines and crosses, and labeling after adaptation is shown with broken lines and circles. 


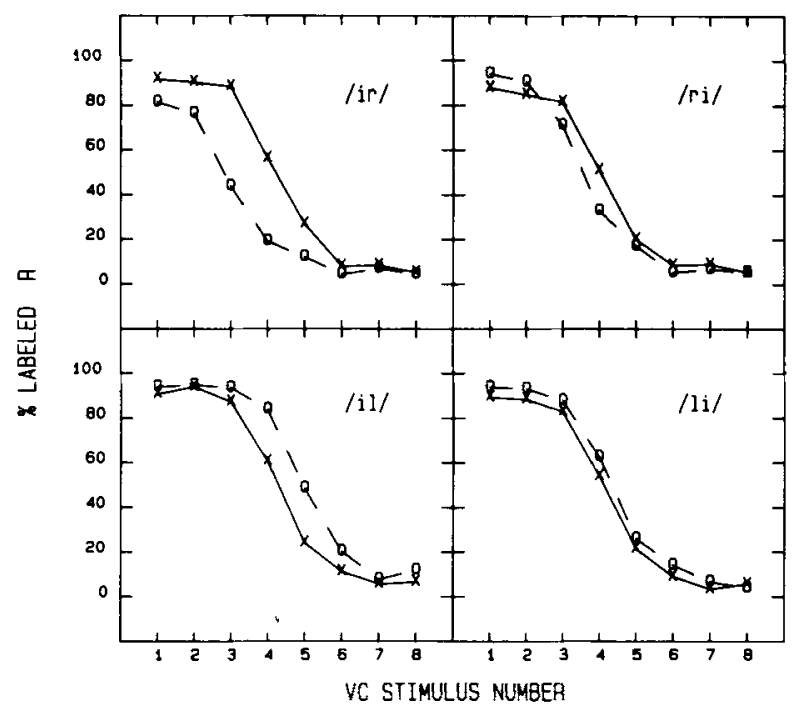

Figure 4. Identification functions for the /ir/-/il/ stimuli, before and after adaptation. Baseline labeling is shown with solid lines and crosses, and labeling after adaptation is shown with broken lines and circles.

report by $10 \%[t(11)=3.82,<.005]$, and $/ \mathrm{li} /$ increased it by $28 \%[t(11)=15.74, p<.001]$. In contrast, the shifts produced by VC adaptors on the /ri/-/li/ continuum were quite small. Report of $/ \mathrm{r} /$ was reduced by only $4 \%$ after adaptation with $/$ ir/ $[t(11)=1.60$, n.s. $] ; /$ il/ adaptation produced a $5 \%$ effect $[t(11)=1.44$, n.s. $]$. The results for the CV test items therefore indicate a strong, though not complete, position-specificity of adaptation.

The results for identification of /ir/-/il/ syllables are similar to those for the CV stimuli. Adaptation of these VC syllables with the /ir/ endpoint was very effective (see Figure 4, bottom left panel); /r/ reports were reduced by $25 \%[t(11)=10.17, p<.001]$. Adaptation with $/ \mathrm{il} /$ was also quite effective, increasing $/ \mathrm{r} /$ report by $16 \%[t(11)$ $=4.78, p<.001]$.

Cross-series effects were much smaller than withinposition ones, though not quite as small as the cross-series effects in the CV case. Adaptation of /ir/-/il// by /ri/ produced a $9 \%$ reduction in $/ \mathrm{r} /$ reports $[t(11)=4.51$, $p<.001]$. The $6 \%$ adaptation effect of $/ \mathrm{li} /$ on the VC series was also reliable $[t(11)=2.23, p<.05]$.

To compare within-position and across-position adaptation effects statistically, a two-way analysis of variance was conducted. In this analysis, difference scores were computed by subtracting average $/ \mathrm{r} / \mathrm{report}$ after /li/ adaptation from such report after/ri/ adaptation (CV adaptors), or after /il/ versus /ir/ adaptation (VC adaptors). This measure reflects the difference in labeling as a function of adaptor. Using the factors test series (CV vs. VC) and adaptors (CV vs. VC), a difference in within- and across-position efficacy would be reflected in an interaction. This interaction was in fact extremely reliable
$[F(1,11)=93.48, p<.001]$. Thus, the average withinposition spread of labeling after $/ \mathrm{r} /$ versus $/ 1 /$ adaptation $(40 \%)$ was reliably greater than the across-position difference $(12 \%)$.

The data from the /r/-/1/ stimuli are generally consistent with those for stop consonants, in that adaptation within position was much stronger than adaptation across syllabic position. In this respect, the data argue against a cancellation explanation of position-specificity, because the $/ r /-/ 1 /$ stimuli do not produce low-level acoustic mismatches of the sort that were illustrated in Figure 1; the large disparity between within-position and across-position effects must instead be attributed to position-specificity of the representations of $/ \mathrm{r} /$ and $/ 1 /$.

Some caution is called for in the interpretation of the data, however. The across-position shifts, though much smaller than the within-position ones, were generally larger than those found in the previous studies that used stop consonant stimuli (Ades, 1974; Miller \& Eimas, 1976; Pisoni \& Tash, 1975; Samuel, Kat, \& Tartter, 1984; Sawusch, 1977b; Wolfe, 1978). The observation of even moderate cross-series effects under conditions that preclude cancellation could be taken as support for the cancellation explanation of the null effects found in previous studies.

A more likely explanation of the observed small crossseries effects is based on the fact that the $/ r /-/ 1 /$ stimuli used here included long $(70-\mathrm{msec})$ consonantal steadystate portions, whose frequency varied across the continuum. These steady-state frequency cues could have served as an invariant (non-position-specific) cue to $/ \mathrm{r} /$ or $/ 1 /$. If so, this low-level acoustic invariance could have produced the observed effects.

In summary, the data argue against the cancellation hpothesis and in favor of the view that syllable-initial and syllable-final consonants are fundamentally different from the perceptual system's perspective. However, the existence of small but reliable across-position effects precludes drawing this conclusion with perfect certainty. In order to provide converging data to allow stronger conclusions, two other experiments were run. In these experiments, different manipulations were used in order to discriminate between the two hypotheses being considered.

\section{EXPERIMENT 2}

The results of Experiment 1 cast doubt on the cancellation hypothesis, and thereby provide support for the notion that there are separate representations of syllableinitial and syllable-final consonants. However, as noted above, the data were not sufficient to completely rule out the cancellation view. Moreover, an adherent of the cancellation position might argue that the $/ r /-/ 1 /$ stimuli are somehow fundamentally dissimilar to the stop consonants used in previous research. Experiment 2 therefore employs stop consonant place-of-articulation stimuli like those used in previous studies that have reported failures 
of cross-position adaptation. Experiment 2 tests whether cross-position effects occur in a paradigm that is similar to selective adaptation: paired contrast.

In the paired-contrast paradigm, listeners identify syllables in much the same way as in the baseline identification task used in Experiment 1. However, each of the test items is immediately preceded by a contextual stimulus, typically one of the test-continuum endpoints. Diehl, Elman, and McCusker (1978) and Diehl, Lang, and Parker (1980) have shown that the labeling pattern obtained with this procedure is similar in many ways to the results obtained with adaptation. At the simplest level, identification is shifted in a contrastive way; an ambiguous stimulus on a $/ \mathrm{ba} /-/ \mathrm{da} /$ continuum will be called $/ \mathrm{ba} /$ if preceded by $/ \mathrm{da} /$, and $/ \mathrm{da} /$ if preceded by $/ \mathrm{ba} /$.

There are various controversies surrounding the relationship of paired contrast and selective adaptation (see, e.g., Diehl, Kluender, \& Parker, 1985, and Sawusch \& Mullenix, 1985). However, the preponderance of evidence indicates that paired-contrast effects involve criterion shifts at a central, complex acoustic level of analysis (see Samuel, 1986; Sawusch \& Jusczyk, 1981). In terms of the cancellation hypothesis, paired contrast should produce effects of phonemic identity, but should be insensitive to the acoustic matching of formant transition structure. As such, if the cancellation hypothesis is correct, cross-series contrast effects should occur; counteracting acoustic shifts should not mask the effects of phonemic matching.

Experiment 2 therefore includes within-position and across-position tests using the paired-contrast paradigm; comparable tests with adaptation are included to provide a replication of previous experiments.

\section{Method}

Stimuli. The stimuli were two eight-step continua, $/ \mathrm{ba} /-/ \mathrm{da} /$ and $/ a b /-/ a d /$, constructed on the Klatt (1980) synthesizer (cascade branch). All syllables were $240 \mathrm{msec}$, including 40 -msec transitions and $200 \mathrm{msec}$ of steady-state vowel. Steady-state frequencies for $F 1, F 2$, and $F 3$ were 740,1240 , and $2545 \mathrm{~Hz}$, respectively. F1 began at $200 \mathrm{~Hz}$ for all CV syllables (and ended at $200 \mathrm{~Hz}$ for all VCs). F2 terminal values ranged from $890 \mathrm{~Hz}(/ \mathrm{b} /)$ to $1590 \mathrm{~Hz}$ $(/ d /)$ in $100-H z$ steps. For F3, these ranged from 2020 to 3070 in $150-\mathrm{Hz}$ steps. F0 was set to $100 \mathrm{~Hz}$ for the first $140 \mathrm{msec}$ of each syllable, and fell linearly to $85 \mathrm{~Hz}$ at syllable offset. The amplitude contour (AV) changed from 55 to $53 \mathrm{~dB}$ during the $40 \mathrm{msec}$ of consonantal transitions and from 53 to $45 \mathrm{~dB}$ during the offset of the vowel (for CVs; for VCs, the contour was reversed); steadystate amplitude during the central $160 \mathrm{msec}$ was $53 \mathrm{~dB}$.

Apparatus and Procedure. Four groups of subjects participated in Experiment 2. Two of the groups identified stimuli from the $/ \mathrm{ba} /-/ \mathrm{da} /$ continuum and two groups identified /ab/-/ad/ stimuli. Similarly, two groups had $/ \mathrm{ba} /$ and $/ \mathrm{da} /$ as adaptors (and context stimuli), and the other two heard /ab/and /ad/ in these roles. Thus, the four groups embodied the factorial crossing of CV/VC adaptors (contrastors) and test series.

Each subject was run in two sessions, with three tests per session. Each session consisted of a baseline identification test, followed by a paired-contrast test, followed by an adaptation test. The identification test included 28 randomizations of the eight $\mathrm{CV}$ or VC stimuli; the first 4 passes were practice and were not scored. The adaptation test included 24 passes through the eight test stimuli.
The adapting blocks and identification blocks were as in Experiment 1 . Half of the subjects in each group had the $/ \mathrm{b} /$ adaptor in their first session and half had the $/ \mathrm{d} /$. The instructions, apparatus, and procedures on the identification and adaptation tests were the same as in Experiment 1.

The contrast test was presented in two halves, with one half given during each session. Each half included 26 randomizations of the eight test stimuli. Half of the test items were preceded by the appropriate /b/ endpoint (/ba/ or /ab/, depending on subject group), and half were preceded by the /d/ endpoint. The context syllable and test syllable were separated by $500 \mathrm{msec}$ of silence. The subjects were informed that they would hear two syllables on each trial and that they were to identify the second syllable by pushing the appropriate button. The first two passes in each session were not scored, leaving a total of $\mathbf{2 4}$ observations for each test stimulus in the context of each of the two endpoint syllables.

Subjects. Sixty subjects participated in Experiment 2. All were native English speakers with no known hearing problems. Twelve of the subjects failed to label the syllables consistently in one or both baseline tests; these subjects were not included in the analyses. The subjects received course credit for their participation.

\section{Results and Discussion}

The data were analyzed as in Experiment 1: the average percentage report of $/ b /$ was computed for each test stimulus for each subject. The group labeling functions for the $/ \mathrm{ba} /-/ \mathrm{da} /$ test stimuli are shown in Figure 5, with the comparable data for $/ \mathrm{ab} /-/ \mathrm{ad} / \mathrm{shown}$ in Figure 6. For the sake of clarity, these figures omit the baseline labeling and show only the adaptation (top) and contrast (bottom) functions.

Consider first the adaptation results for the CV test stimuli (Figure 5, top two panels). As the left panel demonstrates, the identification of CV stimuli was greatly affected by $\mathrm{CV}$ adaptors: report of $/ \mathrm{b} /$ was $30 \%$ lower after adaptation with $/ \mathrm{ba} /$ than after $/ \mathrm{da} /$ adaptation

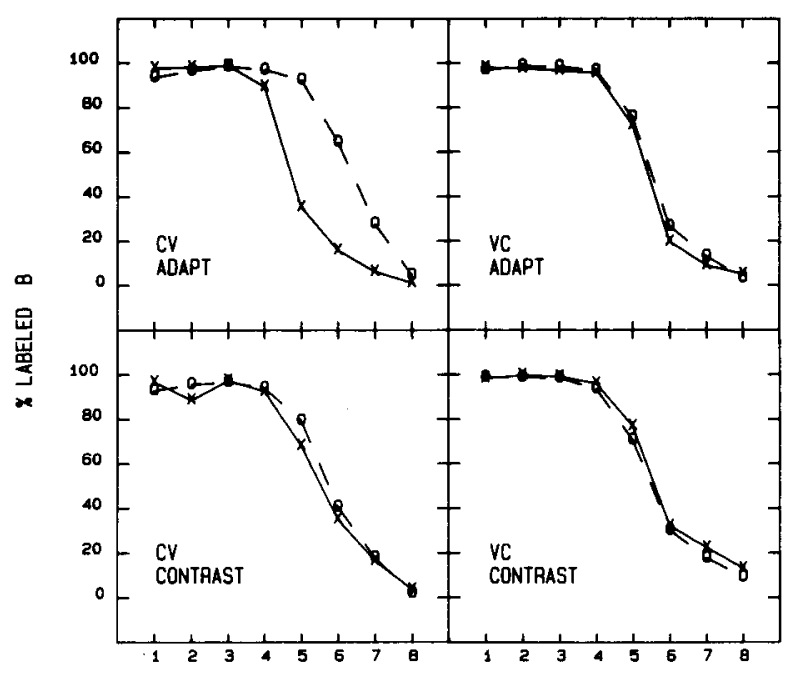

CV STIMULUS NUMBER

Figure 5. Identificaton of the /ba/-/da/ stimuli of Experiment 2, after adaptation, and in the contrast condition. Labeling after adaptation or contrast with $/ b /$ is shown with solid lines and crosses, and the corresponding data from the / $d /$ conditions are shown with broken lines and circles. 


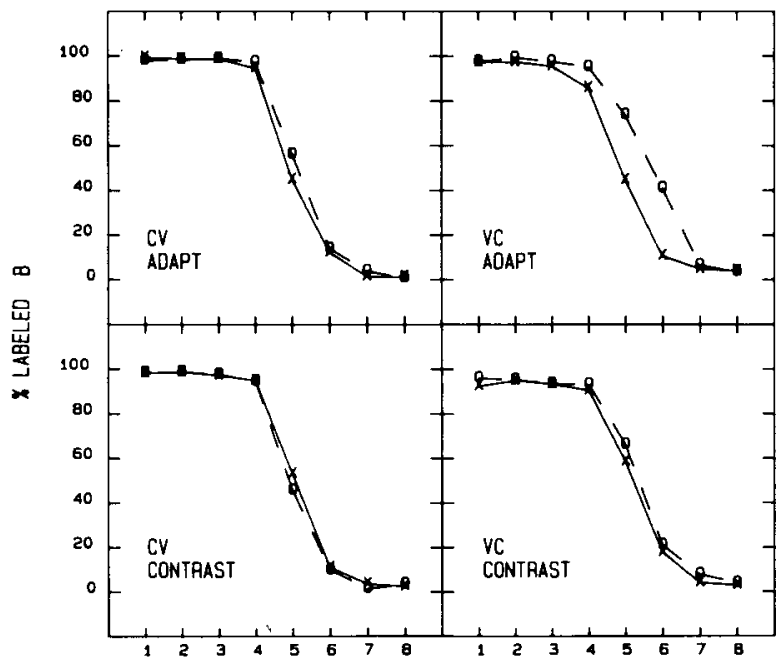

VC STIMULUS NUMBER

Figure 6. Identification of the /ab/-/ad/ stimuli of Experiment 2, before and after adaptation, and in the contrast condition. Labeling after adaptation or contrast with $/ b /$ is shown with solid lines and crosses, and the corresponding data from the / $/ \mathrm{d} /$ conditions are shown with broken lines and circles.

$[t(11)=8.60, p<.001]$. In contrast, the tiny adaptation effect for $\mathrm{VC}$ adaptors suggested in the right-hand panel did not approach significance $[t(11)=0.02$, n.s. $]$. Thus, the adaptation results for the CV test items replicate the position-specificity reported by Ades (1974) and others.

The central issue of Experiment 2 is whether the contrast paradigm, by tapping only higher level processing, produces both within-series and across-series shifts. The data shown in the bottom panels of Figure 5 indicate that no such position independence is to be found. The small shifts (which are typical of the contrast paradigm) are limited to the within-series test: report of /b/ in the context of $/ \mathrm{ba} /$ was $4 \%$ lower than in the context of $/ \mathrm{da} /[t(11)$ $=5.70, p<.04]$; the $\mathrm{VC}$ context stimuli actually produced a small $(3 \%)$, nonsignificant reversal $[t(11)=$ 2.58, n.s.]. Thus, the contrast results for the CV test items do not support a cancellation explanation of the adaptation pattern.

The adaptation results for the VC test items were exactly like those for the CV stimuli. As Figure 6 shows, within-series adaptation (top right panel) produced reliable labeling shifts $[t(11)=6.93, p<.001]$. The $C V$ adaptors did not produce a reliable effect $[t(11)=1.10$, n.s.]. These results, like those for the CV test items, replicate the basic effect of Ades (1974).

The VC contrast data were generally rather weak. The within-series shift of $3 \%$ did not reach significance $[t(11)=1.78$, n.s. $]$. Nor, of course, did the across-series case, with a $2 \%$ reversal observed $[t(11)=2.93$, n.s. $]$.

The results of Experiment 2 may be summarized very simply. The $/ b /-/ d /$ stimuli used in this experiment produced the pattern of adaptation effects and noneffects found by Ades (1974), Miller and Eimas (1976), Pisoni and Tash (1975), Samuel, Kat, and Tartter (1984), Sawusch (1977b), and Wolfe (1978). More importantly, the paired-contrast results paralleled the adaptation data; only within-position context stimuli produced labeling changes. Given that the contrast paradigm produces its effects only at the complex acoustic level, we may conclude that cancellation is not a viable explanation of the failure to find shifts in the cross-position case; there is no low-level acoustic effect contributing to the contrastinduced shifts, and therefore null results cannot be traced to such effects' cancelling opposing shifts at the second level of analysis. Rather, the data from Experiment 2 converge with the preponderance of evidence from Experiment 1: Syllable-initial consonants are represented separately from syllable-final ones.

\section{EXPERIMENT 3}

The first two experiments provide support for the view that the perceptual system differentially processes syllableinitial and syllable-final consonants. Experiment 3 provides a final converging test, which includes both stimuli typical of previous research $(/ \mathrm{b} /-/ \mathrm{d} /)$ and the paradigm used in such studies (selective adaptation). The key to Experiment 3 is the monaural presentation of adaptors and test stimuli. Previous research (e.g., Jamieson \& Cheesman, 1986; Samuel, 1986; Sawusch, 1977a) has suggested that by presenting the adaptor contralaterally to the test syllables, any observed shifts may be attributed to effects at the more abstract, second level of processing.

Note that this manipulation is formally analogous to the use of paired contrast in Experiment 2: contralateral adaptation, like paired contrast, should reveal any effects occurring solely at the more abstract level of processing; no cancellation of effects by acoustic mismatches should occur. This analysis leads to a striking prediction of the cancellation hypothesis: while ipsilateral presentation of adaptor and test items should replicate the standard noneffect across syllabic position, contralateral presentation should produce significant cross-position adaptation. This follows from the fact that under ipsilateral conditions, both first-level simple acoustic factors and second-level complex acoustic factors are present, and (by hypothesis) should cancel. With contralateral presentation, only the higher level is operative - no acoustic cancellation can occur. The cancellation hypothesis thus predicts that ipsilateral adaptation should yield no effect for cross-position stimuli, but that contralateral adaptation should. The position-specificity hypothesis, of course, predicts that because the perceptual system processes syllable-initial consonants and syllable-final ones separately, neither ipsilateral nor contralateral adaptation should be effective across position.

\section{Method}

Stimuli and Apparatus. The stimuli in Experiment 3 were the same $/ \mathrm{ba} /-/ \mathrm{da} / \mathrm{and} / \mathrm{ab} /-/ \mathrm{ad} /$ continua that were used in Experiment 2 . The apparatus was the same as in the first two experiments. 
Procedure. Because the critical prediction involves the crossposition cases, only these conditions were included in the experiment. More specifically, four groups of subjects were run. For two groups, the test series was $/ \mathrm{ba} /-/ \mathrm{da} /$; the adaptor was $/ \mathrm{ab} /$ for one of these and /ad/for the other. For the other two groups, the test continuum was $/ \mathrm{ab} /-/ \mathrm{ad} /$ and the adaptor was either $/ \mathrm{ba} /$ or $/ \mathrm{da} /$.

All subjects participated in a single 1-h test session that included an identification test and an adaptation test. On the identification test, 42 randomizations of the eight test items were presented. Oddnumbered passes were presented to one ear and even-numbered passes were presented to the opposite ear. The first six passes were practice and were not scored. This procedure yielded 18 observations per stimulus per ear.

The adaptation test followed a similar form, with 36 passes alternated between the ears. The other aspects of the adaptation test were as in the first two experiments. On both the identification and adaptation tests, the subjects responded by pushing buttons labeled " $B$ " and "D." The buttons were oriented vertically, and the subjects responded by using two fingers on their dominant hand. This procedure minimized any response-compatibility effects. Half of the subjects in each group were tested with the headphones reversed.

Subjects. Fifty subjects participated in Experiment 3. All were native English speakers with no known hearing problems. Ten subjects failed to label the syllables consistently on one or both baseline identification tests, leaving 10 subjects per condition.

\section{Results and Discussion}

The data were scored and analyzed as in the previous experiments. Figure 7 presents the labeling functions for the CV test series and Figure 8 presents the labeling of the VC stimuli. As the top panels of Figure 7 show, adaptation with /ab/ had no effect whatever on the identification of $/ \mathrm{ba} /-/ \mathrm{da} /$, regardless of whether the testing was ipsilateral or contralateral [larger $t(9)=0.75$, n.s.]. The results for $/ \mathrm{ad} /$, in the bottom panels, were slightly different. Both ipsilateral and contralateral testing produced shifts beween $7 \%$ and $8 \%$, with the ipsilateral case being reliable $[t(9)=4.37, p<.002]$ and the contralateral not

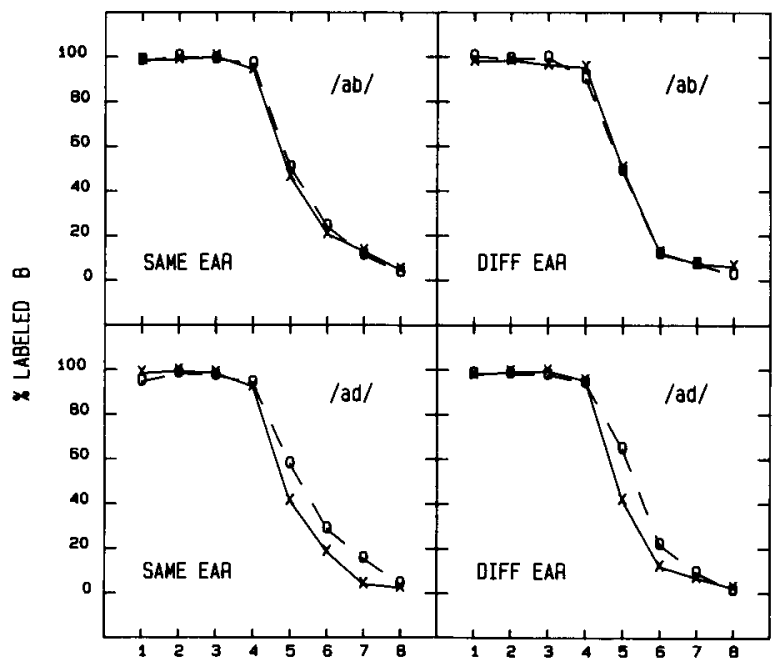

CV STIMULUS NUMBER

Figure 7. Identification of the /ba/-/da/ stimuli of Experiment 3, before and after adaptation. Baseline labeling is shown with solid lines and crosses, and labeling after adaptation is shown with broken lines and circles.

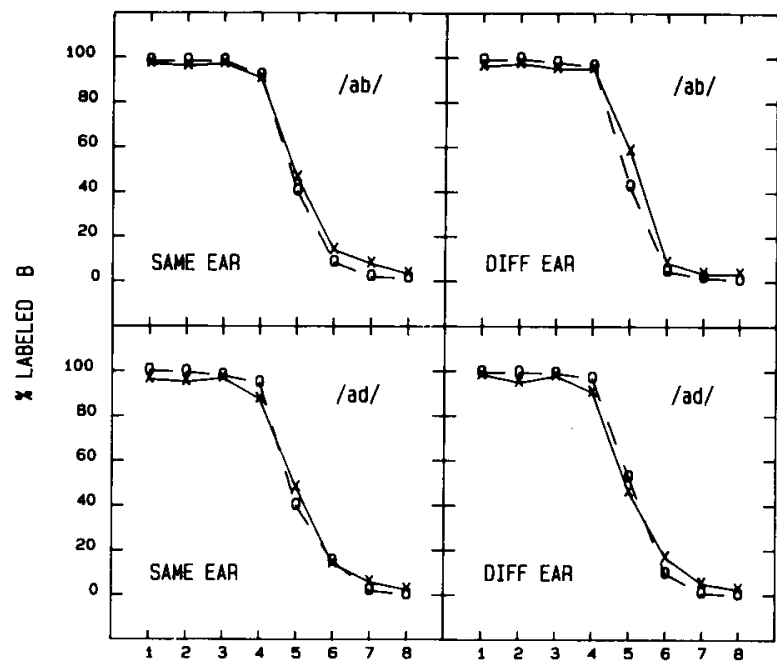

VC STIMULUS NUMBEA

Figure 8. Identification of the /ab/-/ad/ stimuli of Experiment 3, before and after adaptation. Baseline labeling is shown with solid lines and crosses, and labeling after adaptation is shown with broken lines and circles.

$[t(9)=1.61, \mathrm{n} . \mathrm{s}$.$] . The critical issue for Experiment 3$ is whether laterality of adaptation yields different results. As the figure shows, both /ab/ and /ad/ produced comparable patterns as a function of ear $[t(9)=0.13$, n.s.]. Thus, contrary to the cancellation hypothesis, contralateral testing did not permit reliable cross-position adaptation effects to emerge.

The results for the VC test syllables, shown in Figure 8, are even more clear-cut. None of the conditions even approached significance, the closest being ipsilateral $/ \mathrm{ba} /$ 's $4 \%$ reversal $[t(9)=1.36$, n.s. $]$; the other three cases all had $t(9)<1$. Thus, the VC data, like the CV data, give no support whatever to the prediction that contralateral testing would allow the higher level adaptation effect to be seen by eliminating a hypothesized counteracting lower level effect. The results of Experiment 3 are clearly inconsistent with the cancellation hypothesis.

\section{GENERAL DISCUSSION}

The starting point for the present study was a remarkably robust noneffect: adaptation with syllable-initial consonants has no effect on syllable-final ones, and vice versa. Given the ubiquity of adaptation effects, this noneffect is striking. It may well have been the rarity of such a failure of adaptation that led Pisoni and Tash (1975) to suggest that adaptation really was occurring, but that it was not seen because of a counteracting effect. Indeed, their explanation was that there were two adaptation effects; because these effects were in opposite directions, they lead to the observed noneffect, through cancellation.

This cancellation hypothesis is appealing in several ways. It is consistent with the apparent ubiquity of adaptation effects. At a theoretical level, it is consistent with 
the two-level model that has been suggested by a number of authors for a number of reasons (e.g., Fujisaki \& Kawashima, 1969; Pisoni, 1973; Samuel, 1986; Sawusch, 1977a). There is also some empirical support for the occurrence of the kind of acoustically based adaptation required by the cancellation hypothesis (Pisoni \& Tash, 1975).

Despite these virtues, the cancellation explanation does not appear to be corrrect. In the present study, three different methods were used to preempt the occurrence of cancellation. In Experiment 1, this was accomplished by using $/ \mathbf{r} /-/ 1 /$ stimuli that do not produce inverted acoustic-phonetic matches under transformation of syllabic position. In Experiment 2, the approach involved using a paradigm (paired contrast) believed to be unaffected by acoustic detail. Finally, in Experiment 3, putative acoustic cancellation was preempted by the contralateral presentation of adaptors and test items. All three experiments yielded the same result: cross-position effects were small or nonexistent, despite conditions that should have allowed effects to emerge without cancellation by counteracting acoustic adaptation.

If cancellation is not masking underlyingly robust crossposition adaptation, the obvious inference is that consonants in different syllabic positions really do not produce adaptation. This inference has an important implication: if syllabic position determines the occurrence/ nonoccurrence of perceptual effects, then the perceptual system is sensitive to syllabic structure. This conclusion is of some interest in light of the surprising paucity of empirical support for the role of the syllable (at least in English). Aside from the adaptation data, four pieces of evidence have sometimes been offered to support the "psychological reality" of the syllable in perception. First, Savin and Bever (1970) found that subjects were faster at reporting the occurrence of a syllabic target than a phonemic one. Second, using a masking paradigm, Massaro (1972) found the reencoding of the speech signal appears to be done within $250 \mathrm{msec}$. Third, Huggins (1964) reported that interruption of speech (by alternating its presentation between the two ears) was maximally disruptive at a rate that corresponded to the syllabic presentation rate. Finally, Liberman, Shankweiler, Fischer, and Carter (1974) found that very young children were sensitive to the number of syllables in an utterance.

At least two of these four results have been called into question. Foss and Swinney (1973) challenged Savin and Bever's (1970) conclusions, and Norris and Cutler (1988) have recently shown that the syllabic advantage in monitoring times was artifactual. The interruption paradigm of Huggins (1964) was modified by Huggins (1967) and Samuel (1989) to allow a direct test of syllabic effects. In this version, passages are interrupted either on syllabic boundaries or in syllabic middles; the latter should be more disruptive if syllabic interruption underlies the basic effect. Huggins and Samuel both found no difference between the two conditions, making the syllabic interpretation problematic.
Thus, there is at present relatively little evidence to support a perceptual role for the syllable in English. One interesting possibility ties the existing evidence together nicely. If units more like demisyllables (Fujimura, 1976) than standard syllables are used in perceptual processing, then it would not be surprising that evidence for syllables is weak. Note that these units would fit more comfortably within Massaro's (1972) upper bound of $250 \mathrm{msec}$ than full syllables do. Demisyllables also have the virtue of internally dealing with the largest source of noninvariance, since a different demisyllable represents each consonant-vowel and vowel-consonant combination. This feature of such representations is, of course, precisely what is suggested by the adaptation results of the present study, and all of its predecessors: syllable-initial consonants and syllable-final ones must be represented separately.

At this point, there is certainly not sufficient evidence to support a demisyllabic representation with positive certainty. However, such representations are in accord with what is currently known. There is also some recent evidence from a phoneme-monitoring study that is in accord with a demisyllabic interpretation. Cutler, Butterfield, and Williams (1987) found that monitoring times were affected by the structural match between the specification of the target and the actual target: subjects are faster when both are $\mathrm{CV}$ or both are CCV, and slower when there is a mismatch. This is as would be expected if processing is mediated by demisyllables, since $\mathrm{CV}$ and $\mathrm{CCV}$ demisyllables would be distinct. The phoneme-level match would have to be abstracted from the demisyllabic representations.

Clearly, more research is needed to fully determine the set of representations of the speech signal as it goes from its initial auditory code to its final linguistic one. The results of the experiments reported here indicate that in this chain of processing, syllable-initial and syllable-final consonants are represented separately.

\section{REFERENCES}

ADES, A. E. (1974). How phonetic is selective adaptation? Experiments on syllable position and vowel environment. Perception \& Psychophysics, 16, 61-66.

Blakemore, C., \& Sutton, P. (1969). Size adaptation: A new aftereffect. Science, 166, 245-257.

Cutler, A., Butterfield, S., \& Williams, J. N. (1987). The perceptual integrity of syllabic onsets. Journal of Memory \& Language, 26, $406-418$.

Diehl, R. L., Elman, J. L., \& McCusker, S. B. (1978). Contrast effects in stop consonant identification. Joumal of Experimental Psychology: Human Perception \& Performance, 4, 599-609.

Diehl, R. L., KLuender, K. R., \& Parker, E. M. (1985). Are selective adaptation and contrast effects really distinct? Journal of Experimental Psychology: Human Perception \& Performance, 11, 209-220.

Diehl, R. L., LANG, M., \& Parker, E. M. (1980). A further parallel between selective adaptation and response contrast. Journal of Experimental Psychology: Human Perception \& Performance, 6, 24-44.

Eimas, P., \& Corbit, J. (1973). Selective adaptation of linguistic feature detectors. Cognitive Psychology, 4, 99-109.

EIMAS, P. D., \& MiLLER, J. L. (1978). Effects of selective adaptation of speech and visual patterns: Evidence for feature detectors. In H. L. 
Pick \& R. D. Walk (Eds.), Perception and experience (pp. 307-345). New York: Plenum Press.

Foss, D. J., \& SWINNey, D. A. (1973). On the psychological reality of the phoneme: Perception, identification, and consciousness. Journal of Verbal Learning \& Verbal Behavior, 12, 246-257.

Fujimura, O. (1976). Syllables as concatenated demisyllables and affixes. Journal of the Acoustical Society of America, S55

FuJISAKI, H., \& KAWASHIMA, T. (1969). On the modes and mechanisms of speech perception. Annual Report of the Engineering Research Institute, 28, 67-73.

Huggins, A. W. F. (1964). Distortion of the temporal pattern of speech: Interruption and alternation. Journal of the Acoustical Society of America, 36, 1055-1064.

HugGins, A. W. F. (1967). Distortion of the temporal pattern of speech by syllable tied altemation. Language \& Speech, 10, 133-140.

Jamieson, D. G., Cheesman, M. F. (1986). The locus of selective adaptation in speech perception. Journal of Experimental Psychology: Human Perception \& Performance, 12, 286-294.

KAT, D., \& SAmUEL, A. G. (1984). More adaptation of speech by nonspeech. Journal of Experimental Psychology: Human Perception \& Performance, 10, 512-525.

KLATT, D. H. (1980). Software for a cascade-parallel formant synthesizer. Joumal of the Acoustical Society of America, 67, 971-995.

Liberman, I. Y., Shankweiler, D., Fischer, F. W., Carter, B. (1974). Explicit syllable and phoneme segmentation in the young child. Journal of Experimental Child Psychology, 18, 201-212.

Massaro, D. W. (1972). Preperceptual images, processing time, and perceptual units in auditory perception. Psychological Review, 79, 124-145.

McGovern, K., \& Strange, W. (1977). The perception of /r/ and /l/ in syllable-initial and syllable-final position. Perception \& Psychophysics, 21, 162-170.

MILLER, J. L., \& EimAs, P. D. (1976). Studies on the selective tuning of feature detectors for speech. Journal of Phonetics, 4, 119-127.

NorrIs, D., \& CUTLER, A. (1988). The relative accessibility of phonemes and syllables. Perception \& Psychophysics, 43, 541-550.

Pisoni, D. B. (1973). Auditory and phonetic memory codes in the discrimination of consonants and vowels. Perception \& Psychophysics, $13,253-260$.

Pisoni, D. B., TASH, J. (1975). Auditory property detectors and processing place features in stop consonants. Perception \& Psychophysics, 18, 401-408.

SAMUEL, A. G. (1986). Red herring detectors and speech perception: In defense of selective adaptation. Cognitive Psychology, 18, 452-499.

SAmuel, A. G. (1989). Ear alternation effects are not due to syllabic disruption. Unpublished manuscript.

Samuel, A. G., KAT, D., TARTter, V. C. (1984). Which syllable does an intervocalic stop belong to? A selective adaptation study. Journal of the Acoustical Society of America, 76, 1652-1663.

Samuel, A. G., \& NewPort, E. L. (1979). Adaptation of speech by nonspeech: Evidence for complex acoustic cue detectors. Journal of Experimental Psychology: Human Perception \& Performance, 5. 563-578.

SAvin, H. B., Bever, T. G. (1970). The nonperceptual reality of the phoneme. Journal of Verbal Learning \& Verbal Behavior, 9 , 295-302.

SAWUSCH, J. R. (1977a). Peripheral and central processes in selective adaptation of place of articulation in stop consonants. Journal of the Acoustical Society of America, 62, 738-750.

SAwUSCH, J. R. (1977b). Processing of place information in stop consonants. Perception \& Psychophysics, 22, 417-426.

SawusCH, J. R. (1986). Auditory and phonetic coding of speech. In E. Schwab \& H. Nusbaum (Eds.), Pattem recognition by humans and machines (pp. 51-88). Orlando, FL: Academic Press.

SAwusch, J. R., JusczYK, P. (1981). Adaptation and contrast in the perception of voicing. Journal of Experimental Psychology: Human Perception \& Performance, 7, 408-421.

SAWUSCH, J. R., Mullenix, J. W. (1985). When selective adaptation and contrast effects are distinct: A reply to Diehl, Kluender, and Parker. Journal of Experimental Psychology: Human Perception \& Performance, 11, 242-250.

Simon, H. J., \& Studdert-KenNedy, M. (1978). Selective anchoring and adaptation of phonetic and nonphonetic continua. Joumal of the Acoustical Society of America, 64, 1338-1357.

WolfE, C. G. (1978). Perceptual invariance for stop consonants in different positions. Perception \& Psychophysics, 24, 315-326.

(Manuscript received August 4, 1988; accepted for publication November 30,1988 .) 